\title{
Distributed Relay Selection and Power Control for Multiuser Cooperative Communication Networks Using Buyer/Seller Game
}

\author{
Beibei Wang, Zhu Han*, and K. J. Ray Liu \\ Department of Electrical and Computer Engineering and Institute for Systems Research, \\ University of Maryland, College Park, MD 20742, USA \\ ${ }^{*}$ Department of Electrical and Computer Engineering, Boise State University, Boise, ID 83725, USA
}

\begin{abstract}
The performances in cooperative communications depend on careful resource allocation such as relay selection and power control, but traditional centralized resource allocation needs considerable overhead and signaling to exchange the information for channel estimations. In this paper, we propose a distributed buyer/seller game theoretic framework over multiuser cooperative communication networks to stimulate cooperation and improve the system performance. By employing a two-level game to jointly consider the benefits of source nodes as buyers and relay nodes as sellers, the proposed approach not only helps the source smartly find the relays at relatively better locations and buy optimal amount of power from them, but also helps the competing relays maximize their own utilities by asking the reasonable prices. The game is proved to converge to a unique optimal equilibrium. From the simulation results, the relays in good locations can play more important roles in increasing source node's utility, so the source would like to buy more power from these preferred relays. On the other hand, the relays have to set the proper prices to attract the source's buying because of competition from other relays and selections from the source. Moreover, the distributed game resource allocation can achieve comparable performance compared with the centralized one.
\end{abstract}

\section{INTRODUCTION}

Recently, cooperative communication has gained many attentions as an emerging transmit strategy for future wireless networks [1] [2]. The basic idea is that the relay nodes can help the source node's transmission by relaying the replica of the information. The cooperative communications efficiently take advantage of the broadcasting nature of wireless networks, as well as exploit the inherent spatial and multiuser diversities.

The performances in cooperative communications depend on careful resource allocation such as relay selection and power control. In [3], the power allocation was optimized to satisfy the outage probability criterion. The authors in [4] provided analysis on symbol error rate and optimum power allocation for decode and forward cooperation protocol in wireless networks. The energy-efficient broadcast problem in wireless networks has also been considered in [5]. In [6], the authors evaluated cooperative diversity performance when the best relay is chosen according to the average SNR, and the outage probability of relay selection based on the instantaneous SNR. In [7], the authors proposed a distributed relay selection scheme that requires limited network knowledge and is based on instantaneous SNRs. In [8], the relay assignment problem is solved for the multiuser cooperative communications. In [9], the cooperative resource allocation for OFDM is studied. The authors in [10] presented centralized power allocation schemes assuming all relay nodes help and based on this proposed a selection forward protocol to choose only one 'best' relay node to assist transmission, in order to further minimize the system outage behaviors and improve the average throughput.

However, most existing work focuses on resource allocation by means of centralized fashion. This kind of schemes requires that complete channel state information (CSI) or at least channel statistics be available, which brings considerable overhead and signaling of information about channel estimations. For distributed resource allocations, there are two main questions over multiuser cooperative wireless networks: First, among all the distributed nodes, who can help relay and improve the source's link quality better; Second, for the selected relay nodes, how much power they need to transmit.

To answer these two questions, game theory is a natural, flexible and rich tool which studies how the autonomous nodes interact and cooperate with each other. For game theory literature in the wireless networking, in [11], the behaviors of selfish nodes in the case of random access and power control were examined. In [12] static pricing policies for multipleservice networks were proposed to offer the needed incentives for each node to choose the service that best matched its needs, thereby discouraging over-allocation of resources and improving social welfare. In [13] an incentive mechanism to stimulate forwarding in multihop cellular networks was proposed, which was composed of resource delegation to reduce the cost associated with forwarding and pricing-based rewards to create further forwarding incentive. The authors in [14] proposed a pricing game that stimulated cooperation via reimbursements to the relay assuming nodes were selfish and aimed to maximize their own utilities. In [15], the authors employed cooperative game for single-cell OFDMA resource allocation.

In this paper, we employ a buyer/seller (Stackelberg) game [16] to jointly consider the benefits of source nodes and relay nodes in cooperative communications. The game is divided into two levels of hierarchy: the source node plays the buyerlevel game and the relay nodes play the seller-level game. Each player is selfish and wants to maximize its own benefit. Specifically, (1) the source acts as a buyer and aims to get most benefits at the least possible payment. We analyze how many relay nodes would be selected by the source to participate in the sale process after they announced their optimal prices. In addition, we optimize how much service amount (such as power) the source should buy from each relay node. (2) Each relay acts as a seller and aims to earn the payment which can not only cover their forwarding cost but also gain as much extra profit as possible. Each relay node needs to set the optimal price per unit of the service so as to maximize its 
own benefit.

Then we derive the expressions to the proposed game outcomes, prove the concavity of the utility functions, and show that the solution we derived is the unique optimal equilibrium. From the simulations, because of competition from other relays and selections from the source, the relays have to set the proper prices to attract the source's buying so as to optimize their utility values. The source optimally selects the relays and their relaying power, while the relays set the prices that can maximize their utilities. Specifically, relays closer to the source can play a more important role in increasing source's utility, so the source would like to buy more power from these preferred relays. Meanwhile, in order to attract more consumption from the source, the relay might adopt 'low-price, high-market' policy to further increase its utility value. If the total number of available relays increases, the competitions among relays become more severe and the average price the relays ask will decrease. This will result in a larger utility value to the source and a smaller average payment to the relay. We finally show that the distributed game theoretic resource allocation can achieve comparable performance compared with the centralized one [10].

This paper is organized as follows: Section II describes the system model, and formulates the cooperative optimization as a buyer/seller game. We construct distributed implementation of multiuser cooperation transmission, and provide the solutions in Section III. Simulations are shown in Section IV. Finally, Section V concludes the paper.

\section{System Model AND PROBlem Formulation}

In this section, we first derive the expression of the maximal achievable rate/capacity considering multiple relays help the source node's transmission. Then we formulate the optimization problem using a buyer/seller game theoretic framework.

We employ the amplify-and-forward (AF) cooperation protocol [2] as our system model; other protocols can be considered in a similar way. The cooperative transmission consists of two phases.

At phase one, the signal-to-noise ratio (SNR) that results from direct transmission from the source $s$ to the destination $d$ can be expressed by

$$
\Gamma_{s, d}=P_{s} G_{s, d} / \sigma^{2},
$$

where $P_{s}$ represents the transmit power, $G_{s, d}$ is the channel gain, and $\sigma^{2}$ is the noise variance. The rate at the output of direct transmission is

$$
R_{s, d}^{n c}=W \log _{2}\left(1+\Gamma_{s, d}\right) .
$$

Without loss of generality, we assume that the noises in different channels are i.i.d.. We also assume the channels are stable over each power control interval.

At phase two, we consider the SNR at the destination that results from relay $r_{i}$ relaying source $s$ 's data to the destination. By assuming that $X_{s, r_{i}}$ is the broadcasted signal from source $s$ to relay $r_{i}$, the received signal at relay $r_{i}$ is

$$
Y_{s, r_{i}}=\sqrt{P_{s} G_{s, r_{i}}} X_{s, r_{i}}+\eta_{s, r_{i}},
$$

where $\eta_{s, r_{i}} \sim N\left(0, \sigma^{2}\right)$ and $\sigma^{2}$ is the noise variance. Relay $r_{i}$ amplifies $Y_{s, r_{i}}$ and relays it to the destination in which the received signal is

$$
Y_{r_{i}, d}=\sqrt{P_{r_{i}} G_{r_{i}, d}} X_{r_{i}, d}+\eta_{r_{i}, d}
$$

where

$$
X_{r_{i}, d}=Y_{s, r_{i}} /\left|Y_{s, r_{i}}\right|
$$

is the transmitted signal from relay $r_{i}$ to the destination that is normalized to have unit energy. Substituting (3) into (5), we can rewrite (4) as

$$
Y_{r_{i}, d}=\frac{\sqrt{P_{r_{i}} G_{r_{i}, d}}\left(\sqrt{P_{s} G_{s, r_{i}}} X_{s, r_{i}}+\eta_{s, r_{i}}\right)}{\sqrt{P_{s} G_{s, r_{i}}+\sigma^{2}}}+\eta_{r_{i}, d} .
$$

Using (6), the relayed SNR for the source $s$, which is helped by relay $r_{i}$, is given by:

$$
\Gamma_{s, r_{i}, d}=\frac{P_{r_{i}} P_{s} G_{r_{i}, d} G_{s, r_{i}}}{\sigma^{2}\left(P_{r_{i}} G_{r_{i}, d}+P_{s} G_{s, r_{i}}+\sigma^{2}\right)} .
$$

Therefore, by (2) and (7), we have the rate at the output of maximal ratio combining with relay $r_{i}$ helping in $\mathrm{AF}$ as

$$
R_{s, r_{i}, d}=\gamma_{i} W \log _{2}\left(1+\Gamma_{s, d}+\Gamma_{s, r_{i}, d}\right),
$$

with $\gamma_{i}=\frac{1}{2}$ because 2 phases are used for transmission.

If the relays available to help the source at a certain time consist a set, say $L=\left\{r_{1}, \ldots, r_{N}\right\}$, where $N$ is the total number of relays, then

$$
R_{s, r, d}=\gamma_{L} W \log _{2}\left(1+\Gamma_{s, d}+\sum_{r_{i} \in L} \Gamma_{s, r_{i}, d}\right),
$$

where $\gamma_{L}$ is the bandwidth factor. According to different network applications, $\gamma_{L}$ can have different definitions. For the network with limited bandwidth, the bandwidth should be divided for the source and relays. In our case, if $N^{\prime}$ out of $N$ relays are selected by the source, $N^{\prime} \leq N$, then $\gamma_{L}=\frac{1}{N^{\prime}+1}$. For the network that is more energy constrained, $\gamma_{L}$ is set to 1 , which will be adopted in this paper. We will omit the $\gamma_{L}$ in the expression of the source node's utility to be seen in the following.

To explore the cooperative diversity for multiuser system, from (9), two fundamental questions need to be answered: First, which relay nodes should be included; second, what is the optimal power $P_{r_{i}}$. To answer the questions, we employ Stackelberg game for buyers and sellers as the following formulated problem.

(1) Source/Buyer: The source can be modeled as a buyer and it aims to get most benefits at the least possible payment. So the utility function of the source can be defined as

$$
U_{s}=a R_{s, r, d}-M,
$$

where $R_{s, r, d}$ denotes the achievable rate with the relay nodes helping transmission, $a$ denotes the gain per unit of rate achievement at the MRC output, and

$$
M=\sum_{r_{i} \in L} p_{i} P_{r_{i}}=p_{1} P_{r_{1}}+p_{2} P_{r_{2}}+\cdots+p_{N} P_{r_{N}}
$$

represents the total payment paid by the source to the relay nodes. In (11), $p_{i}$ represents the price per unit of power selling from relay node $i$ to the source $s$, and $P_{r_{i}}$ denotes how much 
power the source would like to buy from relay $r_{i}$ when the prices are announced from the relays.

Suppose the relays helping the source consist a set, still denoted by $L=\left\{r_{1}, \cdots, r_{N}\right\}$, then the optimization problem for the source or buyer's game can be formulated as:

$$
\max _{\left\{P_{r_{i}}, M\right\}} U_{s}=a R_{s, r, d}-M \text {, s.t. }\left\{P_{r_{i}}\right\} \geq 0, r_{i} \in L .
$$

(2) Relays/Seller: Each relay $r_{i}$ can be seen as a seller and aims to earn the payment which not only covers their forwarding cost but also gain as much extra profit as possible. We introduce one parameter $c_{i}$, 'the cost of power for relaying data', in our formulation to correctly reflect relays' consideration about whether they can actually get profit by the sale. Then relay $r_{i}$ 's utility function can be defined as

$$
U_{r_{i}}=p_{i} P_{r_{i}}-c_{i} P_{r_{i}}=\left(p_{i}-c_{i}\right) P_{r_{i}}
$$

where $c_{i}$ is the cost per unit of power in relaying data, $p_{i}$ has the same meaning as in (11), and $P_{r_{i}}$ is the source's decision by optimizing $U_{s}$ described in (12). It is obvious that to determine the optimal $p_{i}$ depends not only on each relay's own channel condition to the destination but also on its counterpart relays' prices. So in the sellers' competition, if one relay asks a higher price than what the source expects about it after jointly comparing all relays' prices and their potential contribution to the cooperative transmission rate, the source will buy less from that relay or even disregard that relay. On the other hand, if the price is too low, the profit obtained by (13) will be unnecessarily low. So there is a tradeoff for setting the price.

Then the optimization problem for relay $r_{i}$ or the seller's game is:

$$
\max _{\left\{p_{i}\right\}>0} U_{r_{i}}=\left(p_{i}-c_{i}\right) P_{r_{i}}, \quad \forall i .
$$

If under optimal asking price $p_{i}^{*}$, the resulting utility of $r_{i}$ is such that $U_{r_{i}}^{*} \leq 0$, then $r_{i}$ will quit the seller's game since it can not cover the basic cost by selling power to the source.

Therefore, the ultimate goal of the above game is to decide the optimal pricing $p_{i}$ to maximize relays' profits $U_{r_{i}}$, the actual number of relays who will finally get selected by the source and the corresponding optimal power consumption $P_{r_{i}}$ to maximize $U_{s}$. Notice that the only signaling required to exchange between the source and relays are the price $p_{i}$ and the information about how much power $P_{r_{i}}$ to buy. Consequently, the proposed two-level game approach can have distributed resource allocation for the cooperative communication networks. The outcome of the games will be shown in details in the following section.

\section{ANALYSIS OF BUYER/SELLER GAME}

We will first analyze the buyer/seller game in details and obtain the closed form solutions to the game outcomes. Based on this, we will further prove the solution is the unique optimal equilibrium. Then we design a price update function for relay nodes and prove its convergence to the unique equilibrium. Finally we compare the performance of our distributed scheme with the centralized one.

\section{A. Source/Buyer Level Analysis}

From the definition in (10), we know

$$
\frac{\partial U_{s}}{\partial P_{r_{i}}}=a \frac{\partial R_{s, r, d}}{\partial P_{r_{i}}}-p_{i}, \quad i=1, \cdots, N .
$$

When $P_{r_{j}}=0, j=1, \cdots, N$, if for relay node $r_{i}, p_{i}$ satisfies $p_{i}<a \frac{\partial R_{s, r, d}}{\partial P_{r_{i}}}$, then we have $\frac{\partial U_{s}}{\partial P_{r_{i}}}>0$, meaning the source node will obtain a larger $U_{s}$ by increasing $P_{r_{i}}$.

Then a question is how each relay node $r_{i}$ asks its price $p_{i}$ at the beginning. Since in a distributed implementation, each relay node does not know the other relay nodes' prices, it is possible to first tentatively set $p_{i}=c_{i}$, which makes sure that the utility is not negative. If under these lowest initial prices, the source node would choose not to buy any power from some relay node $r_{i}$, then $r_{i}$ will not participate in the seller-level game because $U_{r_{i}}=0$.

Now assume the number of relays is $N$ and at first the source tentatively choose $P_{r_{i}}=0, i=1, \cdots, N$, if for some relay, say $r_{j}$, it holds that $c_{j} \geq\left.\left(a \frac{\partial R_{s, r, d}}{\partial P_{r_{j}}}\right)\right|_{P_{r_{j}}=0}$, then $r_{j}$ will not be selected by the source and $P_{r_{j}}=0$. For the remaining relays $r_{i}$ 's, which consist a set $L_{h}=\left\{r_{1}, \cdots, r_{N_{0}}\right\}$, by the first order optimality condition, the following equations must hold at the optimal point:

$$
\frac{\partial U_{s}}{\partial P_{r_{i}}}=0, \quad r_{i} \in L_{h} .
$$

Solving (16), we can get its solution in the following lemma.

Lemma 1: The optimal power consumption from each relay node is

$$
P_{r_{i}}^{*}=\sqrt{\frac{A_{i} B_{i}}{p_{i}}} \frac{Y+\sqrt{Y^{2}+4 X W^{\prime}}}{2 X}-B_{i},
$$

where $W^{\prime}=\frac{a W}{\ln 2}, X=1+\sum_{r_{j} \in L_{h}} A_{j}, \quad Y=$ $\sum_{r_{j} \in L_{h}} \sqrt{p_{j} A_{j} B_{j}}, A_{i}=\frac{P_{s} G_{s, r_{i}}}{\left(\sigma^{2}+P_{s} G_{s, d}\right)}$, and $B_{i}=\frac{P_{s} G_{s, r_{i}}+\sigma^{2}}{G_{r_{i}, d}}$. Proof: See Appendix A.

After the source announces the above $P_{r_{i}}^{*}$ to the relays in $L_{h}$, they will gradually increase the prices $p_{i}$ to get possibly more benefit round by round. This will lead the source to buy decreasing amount of $P_{r_{i}}^{*}$. To ensure that the power consumption is feasible/nonnegative, when the source feeds the $P_{r_{i}}^{*}$ to the relays, the expression in (17) should be modified as

$$
\left(P_{r_{i}}\right)^{+}=\max \left(P_{r_{i}}^{*}, 0\right) .
$$

In this way the relay can correspondingly ask a proper price to earn maximal utility instead of being disregarded by the source and the resulting $P_{r_{i}}^{*}$ will be positive.

The solution above can also be verified by Karush-KuhnTucker (KKT) condition [17].

Lemma 2: The optimal power consumption $\left\{\left(P_{r_{i}}\right)^{+}\right\}_{i=1}^{N}$ is a global optimal solution to (12).

\section{Proof: See Appendix B.}

\section{B. Relay/Seller Level Analysis}

Substituting (17) into (14), we have

$$
\max _{\left\{p_{i}\right\}>0} U_{r_{i}}=\left(p_{i}-c_{i}\right) P_{r_{i}}^{*}\left(p_{1}, \ldots, p_{i}, \ldots, p_{N_{0}}\right) .
$$


Note this is a noncooperative game by the relay, and there exists a tradeoff between the price $p_{i}$ and relay's utility $U_{r_{i}}$. If the relay in a better channel condition asks for a relatively lower price $p_{i}$ at first, the source would be glad to buy more power from the cheaper seller and $U_{r_{i}}$ will increase as $p_{i}$ grows. When $p_{i}$ keeps growing and exceeds some certain value, the source would think it is no longer profitable to buy power from the relay even though the relay may be in very good channel condition. In this way $P_{r_{i}}$ will shrink hence result in a decrement of $U_{r_{i}}$. Therefore there is an optimal price for each relay to ask for, depending on the relays' channel conditions. And the optimal price is also affected by other relays' prices since the source only chooses the most beneficial relays among all the relays.

From the analysis above, by the first order optimality condition, it follows that

$$
\frac{\partial U_{r_{i}}}{\partial p_{i}}=P_{r_{i}}^{*}+\left(p_{i}-c_{i}\right) \frac{\partial P_{r_{i}}^{*}}{\partial p_{i}}=0, \quad r_{i} \in L_{h}
$$

Expand (20), we can get the following equation arrays,

$$
\begin{aligned}
& \sqrt{\frac{A_{i} B_{i}}{p_{i}}} \frac{Y+\sqrt{Y^{2}+4 X W^{\prime}}}{2 X}-B_{i}+\left(p_{i}-c_{i}\right) \times \sqrt{\frac{A_{i} B_{i}}{p_{i}}} \\
& \times \frac{Y+\sqrt{Y^{2}+4 X W^{\prime}}}{2 X} \times\left(-\frac{1}{2} \frac{1}{p_{i}}\left(1-\frac{\sqrt{p_{i} A_{i} B_{i}}}{\sqrt{Y^{2}+4 X W^{\prime}}}\right)\right)=0,
\end{aligned}
$$

Solving them for the unknowns $p_{i}$, we have

$$
p_{i}^{*}=p_{i}^{*}\left(\sigma^{2},\left\{G_{s, r_{i}}\right\},\left\{G_{r_{i}, d}\right\}\right), \quad r_{i} \in L_{h} .
$$

The solution (18) and (22) is an equilibrium (Stackelberg Equilibrium), since for each $r_{i} \in L_{h}$, any $\widetilde{p}_{i}$ and any $\tilde{P}_{r_{i}}, U_{r_{i}}\left(p_{1}^{*}, \cdots, \widetilde{p}_{i}, \cdots, p_{r_{N_{0}}}^{*}\right) \leq U_{r_{i}}\left(p_{1}^{*}, \cdots, p_{i}^{*}, \cdots, p_{r_{N_{0}}}^{*}\right)$, where $N_{0}=\left|L_{h}\right|$, and $U_{s}\left(P_{r_{1}}^{+}, \cdots, \tilde{P}_{r_{i}}, \cdots, P_{r_{N}}^{+}\right) \leq$ $U_{s}\left(P_{r_{1}}^{+}, \cdots, P_{r_{i}}^{+}, \cdots, P_{r_{N}}^{+}\right)$, where $N$ is the total number of available relays.

So finally we can get the optimal pricing $p_{i}^{*}$ to maximize relays' utilities $U_{r_{i}}$, the actual number of relays which will get selected by the source and the corresponding optimal power consumption $P_{r_{i}}^{*}$ to maximize $U_{s}$. The convergence of the problem can be proved in a similar way as in [18], and we will give the proof in the following subsections.

\section{Properties of the Equilibrium}

In this subsection, we prove the existence of the Stackelberg Equilibrium (SE) and conditions for the SE to be optimal by the following properties.

Property 1: The utility function of the source $U_{s}$ is concave in $\left\{P_{r_{i}}\right\}_{i=1}^{N}$, where $P_{r_{i}} \geq 0, \forall i$, when each relay's price $p_{i}$ is announced.

Proof: See Appendix C.

Property 2: The optimal power consumption $P_{r_{i}}^{*}$ is decreasing with $p_{i}$ when other relays' prices are some fixed values.

Proof: See Appendix D.

Property 3: The utility function of each relay $U_{r_{i}}$ is concave in its own asking price $p_{i}$, when its power consumption is the optimized purchase amount from the source as calculated in (17) and other relays' prices are some fixed values.

Proof: See Appendix E.
Theorem 1: The $\left\{P_{r_{i}}^{*}\right\}_{i=1}^{N}$ and $\left\{p_{i}^{*}\right\}_{i=1}^{N_{0}}$ solved in Section III-A and III-B is the SE for this buyer/seller game, where SE means the strategy profile that serves best each type of players, given the strategies of the other players.

Proof: See Appendix F.

In practical implementation, the relays will try relatively low asking prices at the beginning, e.g. the $\operatorname{cost} c_{i}$, and then gradually increase the price according to optimal power purchase of the source. As Property 1 shows, the $U_{s}$ is concave in $P_{r_{i}}$, so if all relays' prices finally approach to the optimal values, then the source will also optimally pick the most beneficial power consumptions. Therefore, these iterative procedures will stop until all relays' prices converge to the optimal values to get the maximal utilities, i.e. the SE. In the following we will show the convergence of the relays' prices by constructing a simple update function.

\section{Convergence of Distributed Price Update Function}

From the explanation in the above, the relays will increase their utilities by increasing their prices from reasonable lower values, say $c_{i}$, the cost of power for relaying data, to the optimal ones. That means, $\frac{\partial U_{r_{i}}}{\partial p_{i}}$ changes from positive to zero. Starting from this, we can design an update function for relay's price as follows. In each round of price update until convergence occurs, it holds that

$$
\frac{\partial U_{r_{i}}}{\partial p_{i}}=\frac{\partial}{\partial p_{i}}\left[\left(p_{i}-c_{i}\right) P_{r_{i}}^{*}\right]=P_{r_{i}}^{*}+\left(p_{i}-c_{i}\right) \frac{\partial P_{r_{i}}^{*}}{\partial p_{i}} \geq 0 .
$$

By Property 2, $\frac{\partial P_{r_{i}}^{*}}{\partial p_{i}}<0$, after re-arranging (23), we have

$$
p_{i} \leq I_{i}(\boldsymbol{p}) \triangleq c_{i}-\frac{P_{r_{i}}^{*}}{\partial P_{r_{i}}^{*} / \partial p_{i}}
$$

It's worth noting that the value of $\frac{\partial P_{r_{i}}^{*}}{\partial p_{i}}$ here is negative before $p_{i}$ grows to $\hat{p}_{i}$ such that $P_{r_{i}}^{*}<0$. However, based on utility maximization consideration, the relay will adjust price before $p_{i}$ touches $\hat{p}_{i}$ according to the $\left(P_{r_{i}}\right)^{+}$.

Then the relays' prices update requirements can be described by a vector inequality of the form

$$
\boldsymbol{p} \leq \boldsymbol{I}(\boldsymbol{p}),
$$

where $\boldsymbol{p}=\left(p_{1}, \cdots, p_{N_{0}}\right)$, with $p_{i}$ denoting relay $r_{i}$ 's price; $\boldsymbol{I}(\boldsymbol{p})=\left(I_{1}(\boldsymbol{p}), \cdots, I_{N_{0}}(\boldsymbol{p})\right)$, with $I_{i}(\boldsymbol{p})$ representing the price competition constraint to $r_{i}$ from the other relay nodes. A price vector $\boldsymbol{p} \geq 0$ is feasible if it satisfies the constraints in (25).

For a set $L_{h}$ of $N_{0}$ relays with competition constraints (25), the iterations of the price update algorithm can be expressed as

$$
\boldsymbol{p}(t+1)=\boldsymbol{I}(\boldsymbol{p}(t)) .
$$

We will show next that the convergence of the iteration in (26) by proving $\boldsymbol{I}(\boldsymbol{p})$ is a standard function [18].

Definition: A function $\boldsymbol{I}(\boldsymbol{p})$ is standard if for all $\boldsymbol{p} \geq 0$ the following properties are satisfied [18]:

- Positivity: $\boldsymbol{I}(\boldsymbol{p})>0$,

- Monotonicity: If $\boldsymbol{p} \geq \boldsymbol{p}^{\prime}$, then $\boldsymbol{I}(\boldsymbol{p}) \geq \boldsymbol{I}\left(\boldsymbol{p}^{\prime}\right)$,

- Scalability: For all $\alpha>1, \alpha \boldsymbol{I}(\boldsymbol{p})>\boldsymbol{I}(\alpha \boldsymbol{p})$.

Corollary 1: The price update function $\boldsymbol{I}(\boldsymbol{p})$ is standard. 

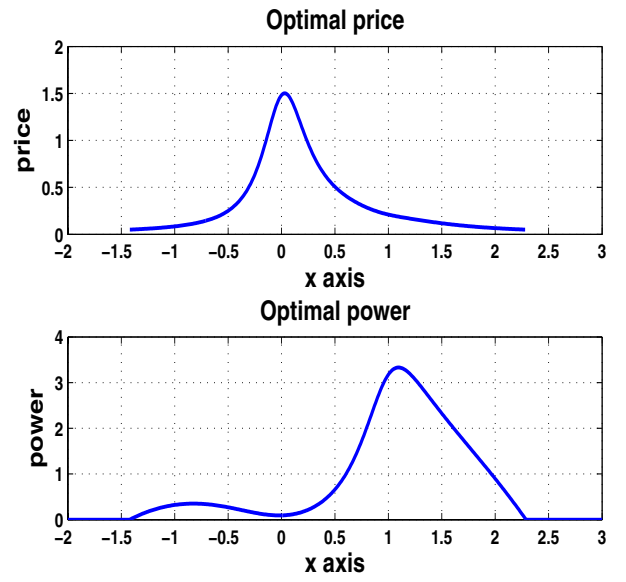

Fig. 1. Optimal price and power of the relay in different locations

Due to limit of space, we will omit detailed proof procedures of Corollary 1 here.

In [18], a proof has been given that starting from any feasible initial power vector $\boldsymbol{p}$, the power vector $\boldsymbol{I}^{n}(\boldsymbol{p})$ produced after $n$ iterations of the standard power control algorithm gradually converges to a unique fixed point. Therefore, we can conclude that starting from a feasible initial price vector $\boldsymbol{c}=\left(c_{1}, \cdots, c_{N_{0}}\right)$, where $c_{i}$ is the cost per unit of power for relay node $r_{i}$, the iteration of the standard price updating produces a non-decreasing sequence of price vectors $\boldsymbol{I}^{n}(\boldsymbol{c})$ that converges to a unique fixed point $\boldsymbol{p}^{*}$.

It is worth mentioning that although the closed-form solutions $\left\{P_{r_{i}}^{*}\right\}_{i=1}^{N}$ in (17) and $\left\{p_{i}^{*}\right\}_{i=1}^{N^{\prime}}$ in (22) are functions of the channel-state information, in practical implementation of the game, the only signallings between the relay nodes and the source node are the instant prices and corresponding powers. Due to Property 1, after the source node first tentatively chooses $P_{r_{i}}=0$, where $i \in L_{h}$, once the relays inform the source of their price information $p_{i}$, the source can find the optimal power $P_{r_{i}}^{*}$ by gradually increasing $P_{r_{i}}$ until $U_{s}$ reaches its maximum. While for each relay, by gradually increasing $p_{i}$ it will get feedback of $P_{r_{i}}^{*}$ and $\partial P_{r_{i}}^{*} / \partial p_{i}$ from the source, then it can update $p_{i}$ using $I_{i}(\boldsymbol{p})$ in (24) until $p_{i}$ converges to $p_{i}^{*}$. Therefore, the proposed game achieves its equilibrium in a distributed way with local information.

\section{E. Comparison with Centralized Optimal Scheme}

In order to compare the performance of our proposed buyer/seller game scheme and the conventional centralized optimal power allocation problem in collaborative communication, we will briefly summarize a centralized problem formulation and give the closed form solutions in this subsection, then we will show the numerical comparison of performances in Section IV. Also here we will mainly focus the performance of the AF protocol and other protocols can be generalized in a similar way. Suppose the system resources are shared by all available $N$ relay nodes, which can help the source transmit data in orthogonal channels, say TDMA. By [2] and [10], we can model the goal as allocating power among relay nodes
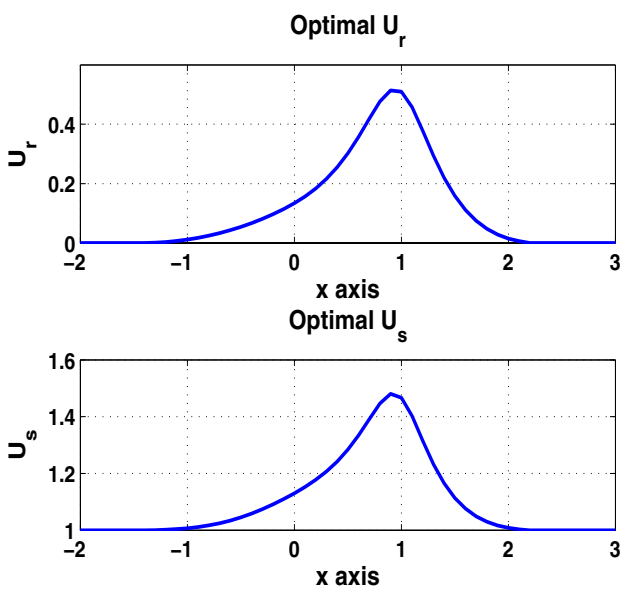

Fig. 2. Optimal utilities of the relay and the source in different locations

to maximize $R_{s, r, d}$ defined in (9) as an optimization problem under both sum and individual power constraints.

$$
\begin{aligned}
\max & \frac{W}{N+1} \log _{2}\left(1+\Gamma_{s, d}+\sum_{r_{i} \in L} \Gamma_{s, r_{i}, d}\right), \\
\text { s.t. } & \sum_{i} P_{r_{i}} \leq P_{r}^{t o t}, 0 \leq P_{r_{i}} \leq P_{r_{i}}^{\max } \quad \forall i .
\end{aligned}
$$

Because $\log _{2}(1+x)$ is a strictly increasing function of $x$, reorganizing the objective function of (27), we can get an equivalent optimization problem as in [10],

$$
\begin{gathered}
\min \sum_{i=1}^{N} \frac{P_{s}^{2} a_{i}^{2}+P_{s} a_{i}}{P_{s} a_{i}+P_{r_{i}} b_{i}+1} \\
\text { s.t. } \quad \sum_{i} P_{r_{i}} \leq P_{r}^{t o t}, 0 \leq P_{r_{i}} \leq P_{r_{i}}^{\max } \quad \forall i,
\end{gathered}
$$

where $a_{i}=\frac{G_{s, r_{i}}}{\sigma^{2}}$ and $b_{i}=\frac{G_{r_{i}, d}}{\sigma^{2}}$.

The solution of (28) can be solved as

$$
P_{r_{i}}=\left(\sqrt{\frac{P_{s}^{2} a_{i}^{2}+P_{s} a_{i}}{b_{i}}} \lambda-\frac{P_{s} a_{i}+1}{b_{i}}\right)_{0}^{P_{r_{i}}^{\max }},
$$

where $\lambda$ is a constant chosen to meet the total power constraint and $(x)_{l}^{u}=l(x<l), x(l \leq x \leq u), u(x>u)$.

However, the centralized optimal power allocation scheme requires that complete channel state information (CSI), i.e., $G_{s, d}, G_{s, r_{i}}$ and $G_{r_{i}, d}$ be available at the destination node to coherently decode the signal. This needs considerable overhead and signaling. In Section IV, we will show our proposed distributed scheme can achieve comparable performance while the needed signaling between source and relays is only the information of price and power consumption amount.

\section{Simulation Results And Analysis}

To evaluate the performances of the proposed scheme and decide what price each relay should ask for and how much power the source should buy from each relay, we performed simulations for multiple relay systems. In what follows, the simulation results for a 1-relay case, for a 2-relay case, and 


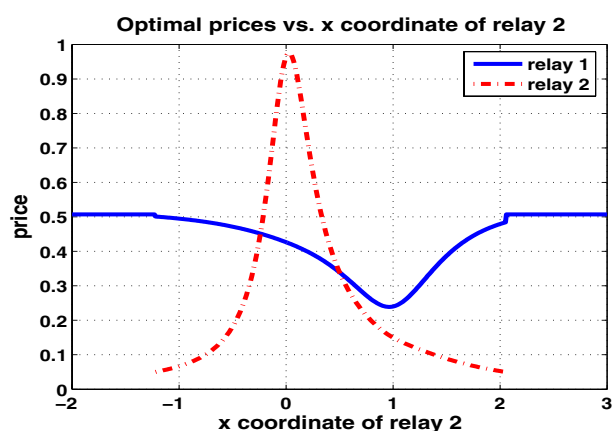

Fig. 3. Optimal prices of two relays when relay 2 moves

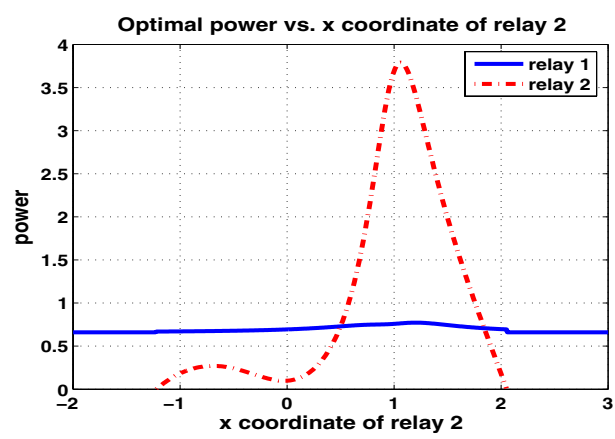

Fig. 4. Optimal power consumptions of two relays when relay 2 moves

for a multiple-relay case are shown. Finally we provided performance comparison of our proposed approach with the centralized optimal scheme.

\section{A. 1-Relay Case}

We set simulations of the first part as follows. There are 1 source-destination pair and 1 relay in the network. The destination was located at coordinate $(0,0)$, and the source was located at coordinate $(1,0)$. We fixed the y coordinate of the relay at 0.25 and the $\mathrm{x}$ coordinate varied within the range of $[-2,3]$. The propagation loss factor was set to 2 . The noise level was $\sigma^{2}=10^{-4}, W=1$, the gain per unit of rate increment $a=1$ and cost per unit of power $c_{i}=0.05, \forall i$.

In Figure 1, we show the optimal price the relay should ask for and the optimal power bought by the source. In this simulation, the relay moves along a line. We observe that when the relay is close to the source at $(1,0)$, it can more efficiently help the source transmit, so the relay would reduce the price to attract the source to buy more service. When the relay moves close to the destination at $(0,0)$, it can use very small amount of power to relay the source's data, so it will set a very high price in order to get more profit by selling this small power. When the relay keeps moving away from the destination, the source would stop buying service because the relay is in such a bad location that asking it to help will be no longer beneficial to the source. Similarly when the relay moves in the opposite direction and locates very far away from the source, the source would not buy service either.

In Figure 2, we show the optimal utility the source and the relay can get using the proposed scheme. When the relay is located close to the source, both the relay and the source can

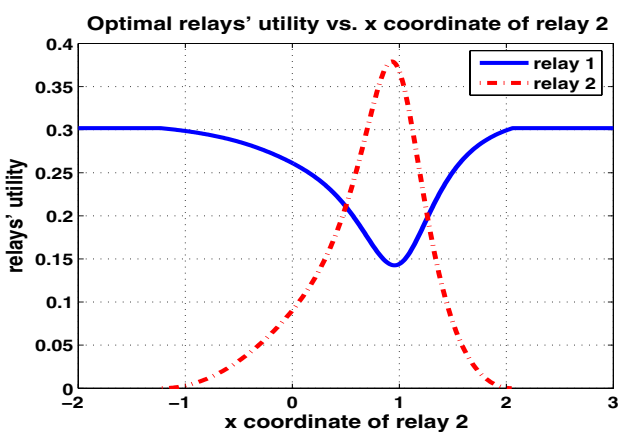

Fig. 5. Optimal utilities of two relays when relay 2 moves

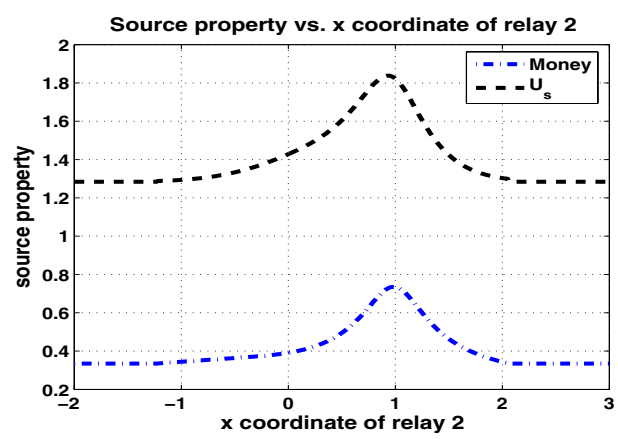

Fig. 6. $U_{s}, M$ when relay 2 moves

get the maximal utility. The reason is that around this location, the relay can most efficiently help the source increase its utility, and the optimal price of the relay is very low compared with that when the relay is at other locations. So the source would like to buy more power, resulting in high utility to the relay. It is worth mentioning that the $\mathrm{x}$-axis represents nodes' channel conditions by using locations, so the questions what price the relay should ask for and how much utility both gameplayers can achieve can be clearly illustrated. If channel effects such as shadowing and fading are considered, the $\mathrm{x}$-axis can be channel conditions and is not related to the distances.

\section{B. 2-Relay Case}

We set up 2-relay simulations to test the proposed scheme. In our simulations, the coordinates of the source and the destination are $(1,0)$ and $(0,0)$ respectively. Relay 1 is fixed at the coordinate $(0.5,0.25)$ and relay 2 moves along the line from $(-2,0.25)$ to $(3,0.25)$. Other settings are the same as the 1-relay case.

In Figure 3 we show the optimal price that each relay should ask to maximize its profit. We can observe that even though only the relay 2 moves, the prices of both relays change accordingly. This fact is because two relays compete and influence each other in the proposed Stackelberg games. When relay 2 is close to the destination at $(0,0)$, it can use very small power to relay the source's information. So relay 2 can set very high price hoping to get more profit by selling small power. When relay 2 is close to the source at $(1,0)$, relay 2 is more suitable to help source transmit. Consequently, in order to attract the source to buy its service, relay 1 has to reduce the price. When relay 2 is faraway, its price will drop 


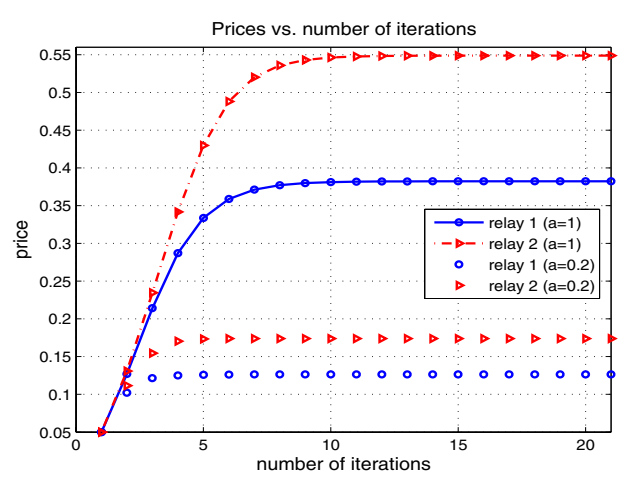

Fig. 7. Prices of relay nodes vs. number of iterations

because it is less competitive compared to relay 1 at location $(0.5,0.25)$. When the utility is less than 0 , relay 2 will quit the competition. At that moment, relay 1 can slightly increase the price since there is no competition. But it cannot increase too much, otherwise relay 2 will rejoin the competition.

As shown in Figure 4, the source will smartly buy different amount of power from the two relays. When relay 2 moves away from the source, $P_{r_{2}}^{*}$ gradually decreases. When relay 2 moves too far away from the source or the destination, the source will not choose relay 2 . When relay 2 is close to the destination, its price shown in Figure 3 is too high, so that the source would not buy much power from relay 2 . When relay 2 quits the competition, relay 1 will increase its price, but the source will buy slightly less. This fact also suppresses the incentive of relay 1 to ask for arbitrarily high price in the absence of competition. Note that when relay 2 moves to $(0.5,0.25)$, the same location as relay 1 , the power consumptions and prices of both relays are the same. This is because the source is indifferent for the two relays locating together and treats them equally.

In Figure 5, we show the optimal utility of two relays. When relay 2 is close to the source, its utility is high, while relay 1 's utility drops. The utility of relay 2 is zero after it quits the competition, while the utility of relay 1 is smooth at the transition points. In Figure 6, we show the optimal utility of the source and the total payment to the relays. When relay 2 is close to the source, the channel conditions are the best in relaying source's data, therefore the relays should get the highest profits and both values reach their maxima.

We also study the convergence of the price update algorithm for a 2-relay case, where relay 2 is at $(0.3,0.25)$ and relay 1 is at $(0.5,0.25)$. In Figure 7 , we can see that when $a=1$, the price updating algorithm converges to the set of the SE with about 12 iterations; when $a=0.2$, the convergence only takes about 6 iterations. The fast convergence of the price updating algorithm shows that the distributed implementation only requires a small overhead in exchanging the price information between the source node and the relay nodes. Also note that a change in the value of the gain factor $a$ does not affect the convergence to the SE, however, the achieved SE is affected. The reason is explained as follows. When $a$ is large, the source node cares the achievable rate more than its payments to the

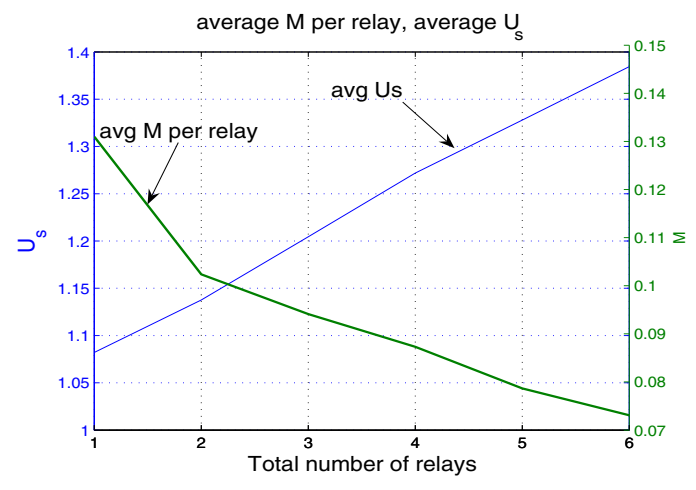

Fig. 8. Optimal $U_{s}$ and average money transfer vs. number of relay nodes

relay nodes, so it likes to buy more power. Thus, the relay nodes can set relatively higher prices to earn more benefits. On the other hand, if $a$ is small, the source node cannot afford high power consumption; hence, the relay nodes can only set relatively lower prices.

\section{Multiple-Relay Case}

We then set up multiple-relay simulations to test the proposed scheme. In these simulations, the coordinates of the source and the destination are $(1,0)$ and $(0,0)$ respectively, and the relays are randomly located within the range of $[-2,3]$ in $x$-axis and $[-2,2]$ in $y$-axis. From Figure 8 , we can observe that as the total number of available relays increases, the source will get a higher utility. However, in this way, the competitions among relays become more severe, which leads to less average payment from the source.

\section{Comparison with Centralized Optimal Scheme}

To compare the performance of the distributed resource allocation scheme with the centralized one, we finally set up simulations as follows. Fix one relay at coordinate $(0.5,0.25)$ and the other at $(0.6,0.25)$ and $(0.4,0.25)$ respectively. As defined in (27), set $P_{r_{i}}^{\max }=1$, and let $P_{r}^{\text {tot }}$ vary within the range of $[0.5,1] \times \sum_{i} P_{r_{i}}^{\max }$. Then we can solve the curve of the maximal achievable rate versus total power consumption constraint. For distributed scheme, by varying $a$, the gain of total achievable rate, we can also get the different total power consumption and corresponding maximal achievable rate. In Figure 9, we can observe that the distributed scheme can achieve approximately the same rate as the centralized optimal power allocation.

\section{CONCLUSiON}

In this paper, we proposed the game theoretic approach for the distributed resource allocation over multiuser cooperative communication networks. We target to answer two questions: who will be the relays and how much power for relaying in the amplified-and-forward cooperative scenario. We employ a buyer/seller (Stackelberg) game to jointly consider the benefits of different types of nodes. The proposed scheme can not only help the source smartly choose relays at better locations but can also help the competing relays ask a reasonable price to maximize their utilities. From the simulation results, relays close to the source can play a more important role in increasing 

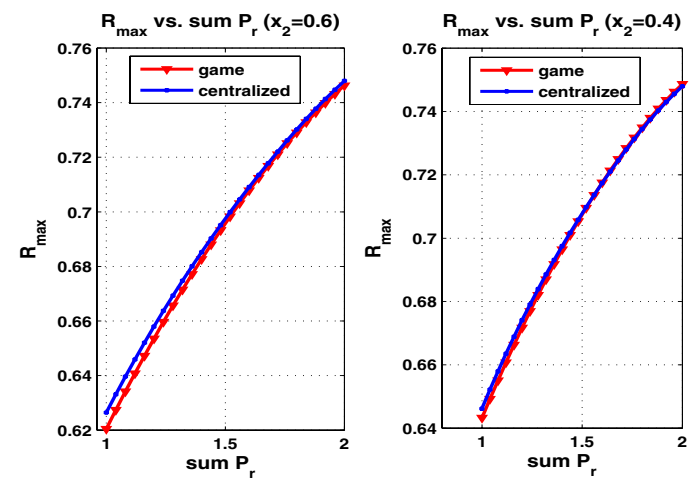

Fig. 9. Optimal achievable rate vs. total power constraint in distributed and centralized schemes

source utility, so the source would like to buy power from these preferred relays. In order to attract more consumption from the source, the relay might adopt 'low-price, high-market' policy to further increase its utility value. If the total number of available relays increases, the source can obtain a larger utility value and the average payment relays can earn will shrink, due to more severe competitions among relay nodes. It's also shown that the distributed resource allocation can achieve comparable performance to the centralized one but with less overhead and signaling of channel estimation information. It is also easy to use the current structures as building blocks in large-scale wireless ad hoc networks to stimulate cooperation among nodes.

\section{A. Proof of Lemma 1}

\section{APPENDIX}

For simplicity, define $C=1+\frac{P_{s} G_{s, d}}{\sigma^{2}}, W^{\prime}=\frac{a W}{\ln 2}$. By (9) and (2) we get the first term of $U_{s}$ when applying $\mathrm{AF}$ protocol as

$$
\begin{aligned}
a R_{s, r, d}= & a W \log _{2}\left(C+\sum_{r_{i} \in L_{h}} \Gamma_{s, r_{i}, d}\right) \\
= & W^{\prime} \ln \left[1+\Delta S N R_{t o t}^{\prime}\right]+W^{\prime} \ln C, \\
& \Delta S N R_{t o t}^{\prime}=\sum_{r_{i} \in L_{h}} \Gamma_{s, r_{i}, d}^{\prime}
\end{aligned}
$$

where

and

$$
\Gamma_{s, r_{i}, d}^{\prime}=\frac{\Gamma_{s, r_{i}, d}}{C}=\frac{A_{i}}{1+\frac{B_{i}}{P_{r_{i}}}}=\frac{A_{i} P_{r_{i}}}{P_{r_{i}}+B_{i}}
$$

with $A_{i}=\frac{P_{s} G_{s, r_{i}}}{\left(\sigma^{2}+P_{s} G_{s, d}\right)}$ and $B_{i}=\frac{P_{s} G_{s, r_{i}}+\sigma^{2}}{G_{r_{i}, d}}$.

Substituting (11) and (30) into (16), we have

$$
\frac{\partial U_{s}}{\partial P_{r_{i}}}=\frac{W^{\prime}}{\left(1+\sum_{r_{k} \in L_{h}} \frac{A_{k} P_{r_{k}}}{P_{r_{k}}+B_{k}}\right)} \frac{A_{i} B_{i}}{\left(P_{r_{i}}+B_{i}\right)^{2}}-p_{i}=0,
$$

i.e.,

$$
\frac{W^{\prime}}{\left(1+\sum_{r_{k} \in L_{h}} \frac{A_{k} P_{r_{k}}+B_{k}}{P_{r_{k}}}\right)}=\frac{p_{i}}{A_{i} B_{i}}\left(P_{r_{i}}+B_{i}\right)^{2} .
$$

Since the left-hand side of (34) is the same for any relay $i$ on the right-hand side, it follows that

$$
\frac{p_{i}}{A_{i} B_{i}}\left(P_{r_{i}}+B_{i}\right)^{2}=\frac{p_{j}}{A_{j} B_{j}}\left(P_{r_{j}}+B_{j}\right)^{2},
$$

then

$$
P_{r_{j}}=\sqrt{\frac{p_{i} A_{j} B_{j}}{p_{j} A_{i} B_{i}}}\left(P_{r_{i}}+B_{i}\right)-B_{j} .
$$

Substitute the above $P_{r_{j}}$ into (32) and simplify, then we have

$$
\Gamma_{s, r_{j}, d}^{\prime}=\frac{A_{j}}{1+\frac{B_{j}}{P_{r_{j}}}}=A_{j}-\sqrt{\frac{p_{j} A_{i} B_{i}}{p_{i} A_{j} B_{j}}} \frac{A_{j} B_{j}}{\left(P_{r_{i}}+B_{i}\right)},
$$

then (31) can be reorganized as

$$
\begin{aligned}
& \triangle S N R_{\text {tot }}^{\prime}=\left[A_{1}-\sqrt{\frac{p_{1} A_{i} B_{i}}{p_{i} A_{1} B_{1}}} \frac{A_{1} B_{1}}{\left(P_{r_{i}}+B_{i}\right)}\right]+\cdots \\
& +\left[A_{i}-\frac{A_{i} B_{i}}{P_{r_{i}}+B_{i}}\right]+\cdots+\left[A_{N_{0}}-\sqrt{\frac{p_{N_{0}} A_{i} B_{i}}{p_{i} A_{N_{0}} B_{N_{0}}}} \frac{A_{N_{0}} B_{N_{0}}}{\left(P_{r_{i}}+B_{i}\right)}\right] \\
& =\sum_{r_{j} \in L_{h}} A_{j}-\sqrt{\frac{A_{i} B_{i}}{p_{i}}} \frac{1}{P_{r_{i}}+B_{i}} \sum_{r_{j} \in L_{h}} \sqrt{p_{j} A_{j} B_{j}} .
\end{aligned}
$$

Substituting (38) into (34), after some manipulation we can have a quadratic equation of $P_{r_{i}}$ as

$$
\begin{aligned}
& \left(1+\sum_{r_{j} \in L_{h}} A_{j}\right)\left[\sqrt{\frac{p_{i}}{A_{i} B_{i}}}\left(P_{r_{i}}+B_{i}\right)\right]^{2} \\
& \quad-\sum_{r_{j} \in L_{h}} \sqrt{p_{j} A_{j} B_{j}}\left[\sqrt{\frac{p_{i}}{A_{i} B_{i}}}\left(P_{r_{i}}+B_{i}\right)\right]-W^{\prime}=0,
\end{aligned}
$$

and its solution is as shown in Lemma 1 .

\section{B. Proof of Lemma 2}

Let $\mathbf{P}_{r}=\left(P_{r_{1}}, \cdots, P_{r_{N}}\right)$, where $N=|L|$ is the number of total available relays, define $f=-U_{s}\left(\mathbf{P}_{r}\right): \mathbf{R}^{N} \rightarrow \mathrm{R}$ and $g_{i}=-P_{r_{i}}: \mathbf{R}^{N} \rightarrow \mathrm{R}$ for $i=1, \cdots, N$. Consider the problem (12) to minimize $f\left(\mathbf{P}_{r}\right)$ subject to $g_{i}\left(\mathbf{P}_{r}\right) \leq 0$ for $i=1, \cdots, N$. As explained by the solution procedures in Section III-A, $\mathbf{P}_{r}^{*}=\left(P_{r_{1}}^{*}, \cdots, P_{r_{N}}^{*}\right)$ is a feasible solution. Define $\nabla=\frac{\partial}{\partial \mathbf{P}_{r}}$, the following equations hold at $\mathbf{P}_{r}^{*}$ :

$$
\nabla f\left(\mathbf{P}_{r}^{*}\right)+\sum_{i=1}^{N} u_{i} \nabla g_{i}\left(\mathbf{P}_{r}^{*}\right)=\mathbf{0},
$$

where $u_{i} g_{i}\left(\mathbf{P}_{r}^{*}\right)=0, u_{i} \geq 0$, for $i=1, \ldots, N$. So $\mathbf{P}_{r}^{*}$ is a KKT solution to (12). Moreover $f$ and $g_{i}$ are differentiable and quasiconvex at $\mathbf{P}_{r}^{*}$, then $\mathbf{P}_{r}^{*}$ is a global optimal solution.

C. Proof of Property 1

Taking the second order derivatives we can get

$$
\begin{aligned}
\frac{\partial^{2} U_{s}}{\partial P_{r_{i}}^{2}}= & -\frac{W^{\prime}}{\left(1+\sum_{k=1}^{N} \frac{A_{k} P_{r_{k}}}{P_{r_{k}}+B_{k}}\right)^{2}}\left(\frac{A_{i} B_{i}}{\left(P_{r_{i}}+B_{i}\right)^{2}}\right)^{2} \\
& -2 \frac{W^{\prime}}{\left(1+\sum_{k=1}^{N} \frac{A_{k} P_{r_{k}}}{P_{r_{k}}+B_{k}}\right)} \frac{A_{i} B_{i}}{\left(P_{r_{i}}+B_{i}\right)^{3}}
\end{aligned}
$$

and

$$
\begin{aligned}
\frac{\partial^{2} U_{s}}{\partial P_{r_{i}} \partial P_{r_{j}}}= & -\frac{W^{\prime}}{\left(1+\sum_{k=1}^{N} \frac{A_{k} P_{r_{k}}}{P_{r_{k}}+B_{k}}\right)^{2}} \\
& \times \frac{A_{i} B_{i}}{\left(P_{r_{i}}+B_{i}\right)^{2}} \frac{A_{j} B_{j}}{\left(P_{r_{j}}+B_{j}\right)^{2}}
\end{aligned}
$$


For each relay, by definition, $W^{\prime}>0, A_{i}>0, B_{i}>0$, also $P_{r_{i}} \geq 0$, so $\frac{\partial^{2} U_{s}}{\partial P_{r_{i}}^{2}}<0$, and $\frac{\partial^{2} U_{s}}{\partial P_{r_{i}} \partial P_{r_{j}}}<0$. It's also straightforward to verify that $\frac{\partial^{2} U_{s}}{\partial P_{r_{i}}^{2}} \frac{\partial^{2} U_{s}}{\partial P_{r_{j}}^{2}}-\left(\frac{\partial^{2} U_{s}}{\partial P_{r_{i}} \partial P_{r_{j}}}\right)^{2}>0$, $\forall i \neq j$. Moreover $U_{s}$ is continuous in $P_{r_{i}}$, so when $P_{r_{i}} \geq 0$, $U_{s}$ is strictly concave in each $\left\{P_{r_{i}}\right\}_{i=1}^{N}$, and jointly concave as well.

\section{Proof of Property 2}

Taking the first order derivative we have

$$
\begin{aligned}
\frac{\partial P_{r_{i}}^{*}}{\partial p_{i}}= & \sqrt{\frac{A_{i} B_{i}}{p_{i}}} \frac{Y+\sqrt{Y^{2}+4 X W^{\prime}}}{2 X} \times \\
& \left(-\frac{1}{2} \frac{1}{p_{i}}\left(1-\frac{\sqrt{p_{i} A_{i} B_{i}}}{\sqrt{Y^{2}+4 X W^{\prime}}}\right)\right)<0,
\end{aligned}
$$

which means $P_{r_{i}}^{*}$ is decreasing with $p_{i}$. This makes sense because when one relay individually increases its price while the others keep the same prices as before, the source will buy less from this relay.

\section{E. Proof of Property 3}

$P_{r_{i}}^{*}$ is a continuous function of $p_{i}$, so $U_{r_{i}}$ is continuous in $p_{i}$ too. Taking derivatives we can get

$$
\begin{aligned}
\frac{\partial U_{r_{i}}}{\partial p_{i}}=- & B_{i}+\sqrt{\frac{A_{i} B_{i}}{p_{i}}} \frac{Y+\sqrt{Y^{2}+4 X W^{\prime}}}{2 X} \times \\
& \left(1-\frac{p_{i}-c_{i}}{2 p_{i}}\left(1-\frac{\sqrt{p_{i} A_{i} B_{i}}}{\sqrt{Y^{2}+4 X W^{\prime}}}\right)\right),
\end{aligned}
$$

and further

$$
\begin{aligned}
\frac{\partial^{2} U_{r_{i}}}{\partial p_{i}^{2}}= & \sqrt{\frac{A_{i} B_{i}}{p_{i}}} \frac{Y_{i}}{2 X}\left(1-\frac{\sqrt{p_{i} A_{i} B_{i}}}{\sqrt{Y^{2}+4 X W^{\prime}}}\right)\left(\frac{-p_{i}-3 c_{i}}{4 p_{i}^{2}}\right) \\
+ & \frac{\sqrt{\frac{A_{i} B_{i}}{p_{i}}}}{8 X p_{i}^{2}\left(\sqrt{Y^{2}+4 X W^{\prime}}\right)^{3}} \\
\times & {\left[\left(Y_{i}^{2}+2 Y_{i} \sqrt{p_{i} A_{i} B_{i}}+4 X W^{\prime}\right)^{2}\left(-p_{i}-3 c_{i}\right)\right.} \\
& +p_{i} A_{i} B_{i}\left(Y_{i}^{2}+2 Y_{i} \sqrt{p_{i} A_{i} B_{i}}\right)\left(-p_{i}-3 c_{i}\right) \\
& \left.+p_{i} A_{i} B_{i} 4 X W^{\prime}\left(-4 c_{i}\right)\right],
\end{aligned}
$$

where $Y_{i}=Y-\sqrt{p_{i} A_{i} B_{i}}$. Since $A_{i}, B_{i}, p_{i}, Y_{i}, c_{i}, X, W^{\prime}>0$, $\frac{\partial^{2} U_{r_{i}}}{\partial p_{i}^{2}}<0$. So $U_{r_{i}}$ is concave with respect to $p_{i}$.

\section{F. Proof of Theorem 1}

When the relays feedback their decision variables $\left\{p_{i}\right\}_{i=1}^{N}$ to the source, by the procedures described in Section III-A, Lemma 1 and Property $1, U_{s}\left(\left\{P_{r_{i}}^{*}\right\}_{i=1}^{N}\right) \geq U_{s}\left(\left\{P_{r_{i}}\right\}_{i=1}^{N}\right)$, i.e., $\left\{P_{r_{i}}^{*}\right\}_{i=1}^{N}$ is the optimal response strategy for the source. From the analysis in Property 3, if relay $i$ gets selected by the source, by the concavity of $U_{r_{i}}$, it can always find its optimal price $p_{i}^{*} \in\left(c_{i}, \infty\right)$, and in this case $U_{r_{i}}>0$. While it locates badly, the source will not choose relay $i$ to help, so actually in this case $p_{i}$ will not affect the source's decision and $U_{r_{i}}=0$. Therefore, at the optimal point $p_{i}^{*}, U_{r_{i}}\left(p_{i}^{*}\right) \geq U_{r_{i}}\left(p_{i}\right)$. So the following equations hold

$$
\begin{gathered}
U_{s}^{*}\left(\left\{P_{r_{i}}^{*}\right\}\right)=\sup _{\left\{P_{r_{i}}\right\} \geq \mathbf{0}} U_{s}\left(\left\{P_{r_{i}}\right\}\right), \\
U_{r_{i}}^{*}\left(p_{i}^{*}\right)=\sup _{p_{i}>c_{i}} U_{r_{i}}\left(p_{i}\right), \quad r_{i} \in L_{h},
\end{gathered}
$$

meaning the $\left\{P_{r_{i}}^{*}, p_{i}^{*}\right\}$ is the the $\mathrm{SE}$ in the buyer/seller game.

\section{REFERENCES}

[1] A. Sendonaris, E. Erkip, and B. Aazhang, "User cooperation diversity, Part I: System description," IEEE Transactions on Communications, vol. 51, no. 11, pp. 1927-1938, Nov. 2003.

[2] J. N. Laneman, D. N. C. Tse, and G. W. Wornell, "Cooperative diversity in wireless networks: efficient protocols and outage behavior," IEEE Transactions on Information Theory, vol. 50, no. 12, pp. 3062-3080, Dec. 2004.

[3] M. O. Hasna and M.-S. Alouini, "Optimal power allocation for relayed transmissions over Rayleigh fading channels," in Proc. IEEE Vehicular Technology Conference, vol. 4, pp. 2461-2465, Jeju, Korea, Apr. 2003.

[4] W. Su, A. K. Sadek, and K. J. R. Liu, "SER performance analysis and optimal power allocation for decode-and-forward cooperation protocol in wireless networks," in Proc. of IEEE Wireless Communications and Networking Conference, pp. 984-989, New Orleans, LA, Mar. 2005.

[5] I. Maric and R. D. Yates, "Cooperative multihop broadcast for wireless networks," IEEE Journal on Selected Areas in Communications, vol. 22, no. 6, pp. 1080-1088, Aug. 2004.

[6] J. Luo, R. S. Blum, L. J. Greenstein, L. J. Cimini, and A. M. Haimovich, "New approaches for cooperative use of multiple antennas in ad hoc wireless neworks," in Proc. IEEE Vehicular Technology Conference, vol. 4, pp. 2769-2773, Los Angeles, CA, Sep. 2004.

[7] A. Bletsas, A. Lippman, and D. P. Reed, "A simple distributed method for relay selection in cooperative diversity wireless networks, based on reciprocity and channel measurements", in Proc. IEEE Vehicular Technology Conference, vol. 3, pp. 1484-1488, Stockholm, Sweden, May 2005.

[8] A. K. Sadek, Z. Han, and K. J. R. Liu, "An efficient cooperation protocol to extend coverage area in cellular networks", in Proc. IEEE Wireless Communications and Networking Conference, vol. 3, pp. 1687-1692, Las Vegas, NV, Apr. 2006.

[9] Z. Han, T. Himsoon, W. Siriwongpairat, and K. J. R. Liu, "Energy efficient cooperative transmission over multiuser OFDM networks: who helps whom and how to cooperate", in Proc. IEEE Wireless Communications and Networking Conference, vol. 2, pp. 1030-1035, New Orleans, LA, Mar. 2005.

[10] Y. Zhao, R. S. Adve, and T. J. Lim, "Improving amplify-and-forward relay networks: optimal power allocation versus selection", in Proc. IEEE International Symposium on Information Theory, Seattle, WA, Jul. 2006.

[11] A. B. MacKenzie and S. B. Wicker, "Game theory and the design of self-configuring, adaptive wireless networks," IEEE Communications Magazine, vol. 39, no. 11, pp. 126-131, Nov. 2001.

[12] L. A. DaSilva, D. W. Petr, and N. Akar, "Static pricing and quality of service in multiple service networks," in Proc. 5th Joint Conference on Information Sciences, vol. 1, pp. 355-358, Atlantic City, MD, Feb. 2000.

[13] M. Lindstrom and P. Lungaro, "Resource delegation and rewards to stimulate forwarding in multihop cellular networks," Proc. IEEE Vehicular Technology Conference, vol. 4, pp. 2152-2156, May 2005.

[14] N. Shastry and R. S. Adve, "Stimulating cooperative diversity in wireless ad hoc networks through pricing", in Proc. IEEE International Conference on Communications, vol. 8, pp. 3747-3752, Istanbul, Turkey, Jun. 2006.

[15] Z. Han, Z. Ji, and K. J. R. Liu, "Fair multiuser channel allocation for OFDMA networks using Nash bargaining and coalitions", IEEE Transactions on Communications, vol. 53, no. 8, pp. 1366-1376, Aug. 2005.

[16] Wikipedia, http ://en.wikipedia.org/wiki/Stackelberg_game

[17] M. S. Barzaraa, Nonlinear programming: theory and algorithms, 2nd ed., John Wiley \& Sons, 1993.

[18] R. Yates, "A framework for uplink power control in cellular radio systems", IEEE Journal on Selected Areas in Communications, vol. 13, no. 7, pp. 1341-1348, Sep. 1995. 